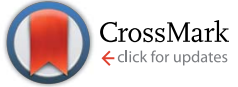

Cite this: RSC Adv., 2017, 7, 14805

Received 23rd December 2016 Accepted 27th February 2017

DOI: $10.1039 / c 6 r a 28600 c$

rsc.li/rsc-advances

\section{Hyaluronate modified upconversion nanoparticles for near infrared light-triggered on-off tattoo systems $\uparrow$}

\author{
Seulgi Han, ${ }^{a}$ Songeun Beack, ${ }^{a}$ Sanghwa Jeong, ${ }^{b}$ Byung Woo Hwang, ${ }^{a}$ \\ Myeong Hwan Shin, ${ }^{a}$ Hyemin Kim, ${ }^{a}$ Sungjee Kim ${ }^{b}$ and Sei Kwang Hahn*a
}

An in vivo on-off tattoo system was developed using upconversion nanoparticles conjugated with hyaluronate (HA-UCNPs). Two-photon microscopy clearly visualized the transdermal delivery of HA-UCNPs into the deep skin tissue. Upon near-infrared light irradiation, invisible HA-UCNPs in the skin were visualized showing the feasibility as a new on-off tattoo system.
Upconversion nanoparticles (UCNPs) doped with lanthanide ions are well-known as promising light delivery materials due to their ability to convert near-infrared (NIR) light to visible light. We can make UCNP to emit dynamically fine-tuned full colour light by adjusting the composition and the pulse-type of NIR laser. NIR light has a relatively high penetration depth compared with visible light because most biomolecules have low absorbance in the NIR window $(700-1100 \mathrm{~nm}) .{ }^{1}$ The long wavelength NIR light is also biocompatible for the tissue due to their low energy. ${ }^{2}$ Along with the remarkable optical excitation and emission properties, and the absence of auto-fluorescence in biological tissues under NIR light excitation, ${ }^{3,4}$ UCNP systems have emerged as a light delivery nanoplatform for photodynamic therapy, photothermal therapy and various photomedicines. It is a crucial issue to achieve non-invasive and controllable light delivery into the deep tissue.

In order for transdermal delivery, UCNP was conjugated with hyaluronate (HA) which has been widely used for a variety of biomedical applications. Recently, HA has been exploited as an effective transdermal delivery carrier of nanomaterials and biopharmaceuticals. ${ }^{5} \mathrm{HA}$ is hygroscopic, but has a hydrophobic patch domain on the molecular backbone. ${ }^{6}$ Consequently, HA facilitates hydrating stratum corneum (SC) composed of the lipid bilayer and enhancing the penetration of tethered payloads through the skin. This outstanding feature of HA might enable the effective transdermal delivery of human growth hormone $(\mathrm{hGH})^{7}$ and nanomaterials like nano graphene oxide ${ }^{8}$ and gold ( $\mathrm{Au}$ ) nanoparticles. ${ }^{9}$ After topical delivery, two-photon microscopy clearly visualized the

${ }^{a}$ Department of Materials Science and Engineering, Pohang University of Science and Technology (POSTECH), 77 Cheongam-ro, Nam-gu, Pohang, Kyungbuk 790-784, Korea.E-mail: skhanb@postech.ac.kr

${ }^{b}$ Department of Chemistry, Pohang University of Science and Technology (POSTECH), 77 Cheongam-ro, Nam-gu, Pohang, Kyungbuk 790-784, Korea

$\dagger$ Electronic supplementary information (ESI) available: Experimental details, Fig. S1, S2 and Table S1. See DOI: 10.1039/c6ra28600c penetration of FITC-labelled HA-hGH conjugate into skin tissues of mice. Furthermore, Au nanorods could be transdermally delivered for theranostic applications. ${ }^{9}$ From the results, HA appeared to be used as a feasible transdermal delivery carrier of nanomaterials with greatly improved compliance.

Here, we developed HA modified UCNP for biocompatible transdermal light delivery. UCNP of $\mathrm{NaYF}_{4}: \mathrm{Yb} / \mathrm{Er}(\mathrm{Y}: \mathrm{Yb}: \mathrm{Er}=$ $69: 28: 3$ ) was synthesized by hydrothermal decomposition method as reported elsewhere. ${ }^{10}$ HA-UCNP could be used as a new kind of on-off tattoo system which can be visualized in a controlled manner by irradiating NIR laser. Scheme 1a shows the simple steps of on-off tattoo system. First, HA-UCNP was synthesized and delivered on the dorsal skin of balb/c nude mice in a desired shape. After transdermal delivery for $40 \mathrm{~min}$, the area loaded with HA-UCNP was cleaned by washing and visualized by the fluorescence of specific shapes after irradiating $980 \mathrm{~nm}$ laser. As shown in Scheme 1b, UCNP was surfacemodified with poly(allylamine) (PAAm) and then conjugated with HA using the EDC/sulfo-NHS chemistry. Oleic acids on the surface of UCNP were replaced with PAAm by the ligand exchange to prepare UCNP-PAAm with amine groups. ${ }^{11}$

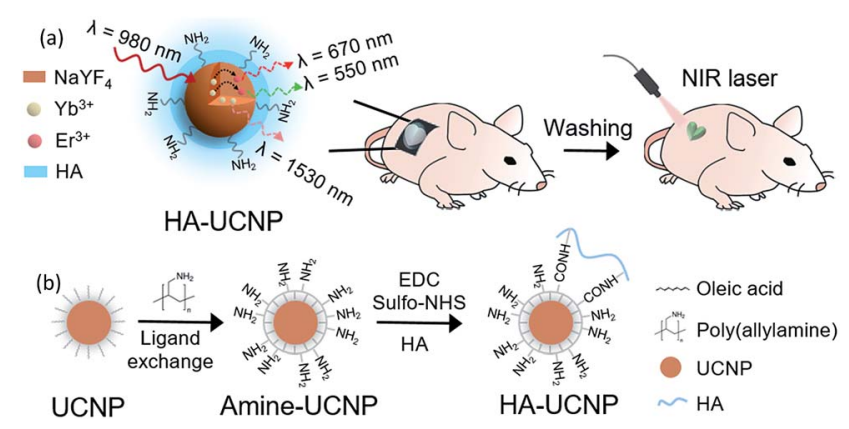

Scheme 1 (a) Schematic illustration for on-off tattoo system using HA-UCNP and NIR laser, and (b) synthetic chemistry of HA-UCNP. 
HA-UCNP was characterized by transmission electron microscopy (TEM), high resolution transmission electron microscopy (HRTEM), dynamic light scattering (DLS), Fourier transform-infrared spectroscopy (FT-IR) and biocompatibility test (Fig. 1 and S1, ESI $\dagger$ ). The core of UCNP capped with oleic acid was showed by TEM with a regular size distribution (Fig. 1b). The uniform crystal lattice of UCNP was confirmed by HRTEM and diffraction pattern analysis. According to HRTEM images (Fig. 1c), the distance of lattice fringes was $0.515 \mathrm{~nm}$, allocating (100) hexagonal crystal plane. The diffraction pattern of UCNP indicated some bright rings of the interplanar spacing for the hexagonal phase (Fig. 1d). Inductively coupled plasma atomic emission spectroscopy (ICP-AES) after preconditioning revealed the composition of $\mathrm{NaYF}_{4}: \mathrm{Yb} / \mathrm{Er}^{12,13}$ (Table S1, ESI $\dagger$ ). The successful synthesis of UCNP-PAAm and HA-UCNP was also (a)

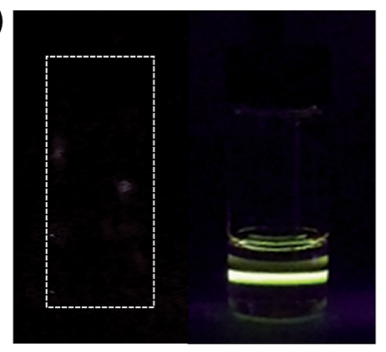

(c)

(e)
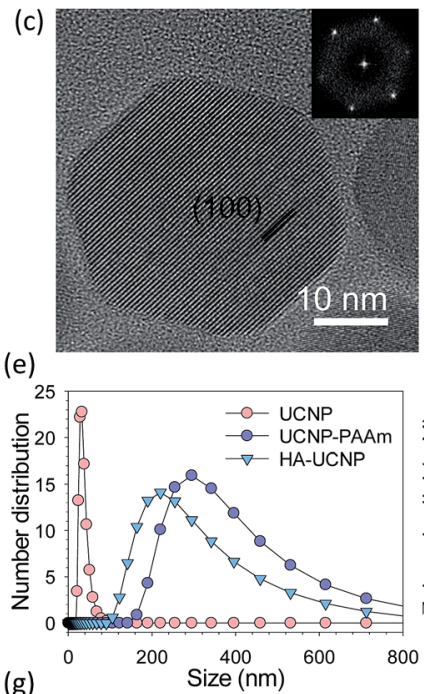

(g)

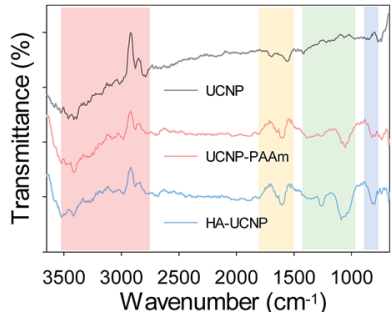

(b)

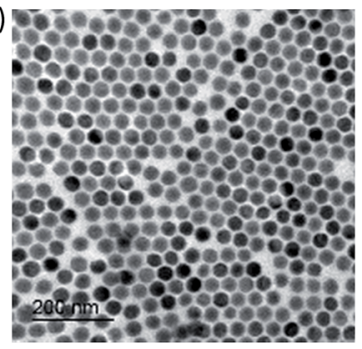

(d)

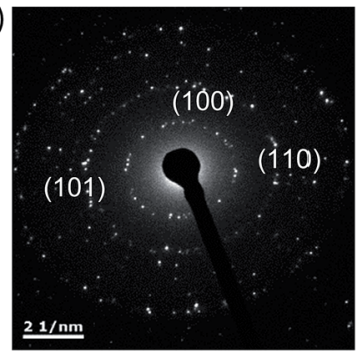

(f)
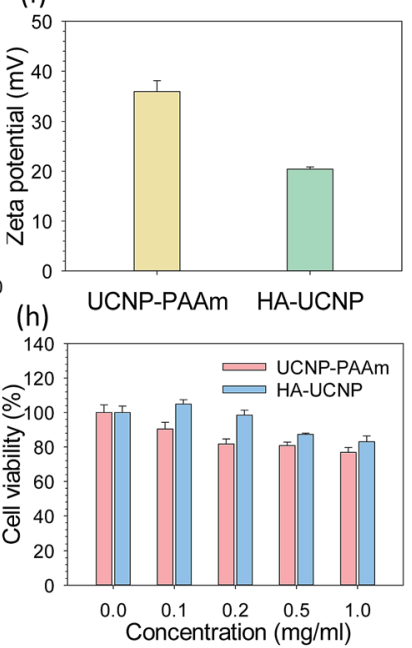

Fig. 1 (a) Photographs of UCNP (NaYF 4 :Yb/Er) fluorescence. (b) TEM images of UCNP cores with a uniform particle size of ca. $30 \mathrm{~nm}$. (c) HRTEM of hexagonal phase UCNP. (d) Diffraction pattern of UCNP. (e) Particle size distribution and ( $f$ ) zeta potential of UCNP-PAAm and HAUCNP. (g) FT-IR spectra of UCNP in cyclohexane, UCNP-PAAm, and HA-UCNP dissolved in DI water. (h) Biocompatibility of UCNP-PAAm and HA-UCNP. confirmed by measuring the size and the zeta potential of nanoparticles. The core particle diameter of UCNP-PAAm and HA-UCNP was maintained as $30 \pm 4 \mathrm{~nm}$. After surface-coating of UCNP with PAAm, the size of modified UCNP increased to $302.87 \mathrm{~nm}$ at the peak value. After conjugation of UCNP-PAAm with HA, the particle size of HA-UCNP decreased to $221.59 \mathrm{~nm}$ (Fig. 1e). It was supposed that polyanionic HA (MW $=100 \mathrm{kDa})$ with a dimension of $c a .250 \mathrm{~nm}$ formed an electrostatic complex with positively charged PAAm on the UCNP. The zeta potential of UCNP-PAAm $(35.98 \pm 2.72 \mathrm{mV})$ decreased to $20.42 \pm 0.38 \mathrm{mV}$ after conjugation with HA (Fig. 1f). Moreover, FT-IR analysis revealed the chemical structural characteristics of HA-UCNP (Fig. 1g). The change at $3300-3600 \mathrm{~cm}^{-1}$ indicated the ligand exchange from oleic acid to PAAm and the change below 1500 $\mathrm{cm}^{-1}$ indicated covalent bonding between the carboxyl group of HA and the amine group of UCNP-PAAm. To verify the conjugation of HA with UCNP-PAAm, HiLyte ${ }^{\mathrm{TM}}$ fluor 647 amines were conjugated to carboxyl groups of HA by the EDC chemistry (Fig. S2, ESI $\dagger$ ). ${ }^{14}$ After that, $40 \%$ of HiLyte-HA was bound to UCNP-PAAm, resulting in $c a$. 200 of HA per the single UCNPPAAm. The cell viability after incubation with UCNP-PAAm and HA-UCNP was assessed by MTT assay (Fig. 1h). NIH3T3 fibroblast cell line was used for the biocompatibility test of UCNP-PAAm and HA-UCNP. As the concentration increased, the cell viability after incubation with UCNP-PAAm appeared to decrease possibly due to the positive charge of samples. The cell viability was $\mathrm{ca} .78 \%$ at the concentration of $1 \mathrm{mg} \mathrm{ml}{ }^{-1}$. On the other hand, the cell viability after incubation with HA-UCNP was higher in the whole range of concentrations. The polyanionic HA might reduce the positive charge of PAAm on UCNP, thereby enhancing the biocompatibility of HA-UCNP.

The fluorescence of UCNP is caused by energy transfer between multiple energy levels of lanthanide ions in the localized $4 \mathrm{f}$ orbitals. UCNP doped with ytterbium $\left(\mathrm{Yb}^{3+}\right)$ as a sensitizer and erbium ion $\left(\mathrm{Er}^{3+}\right)$ as an activator emits red $(\sim 660 \mathrm{~nm})$ and green $(\sim 550 \mathrm{~nm})$ fluorescence at the same time. ${ }^{15,16}$ The ratio between green and red fluorescence could be controlled by changing the intensity of $980 \mathrm{~nm}$ laser (Fig. 2). Accordingly, the colour of fluorescence was changed from green to yellow. It can be explained that the triple electron transfer makes red fluorescence and the quadrupole electron transfer makes green fluorescence. In general, the colour of fluorescence dyes is (a)

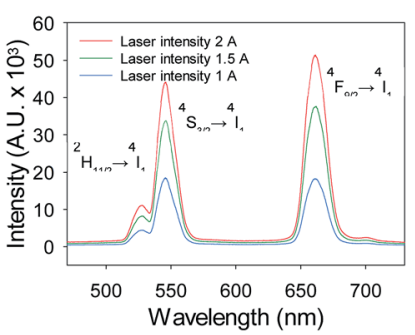

(b)

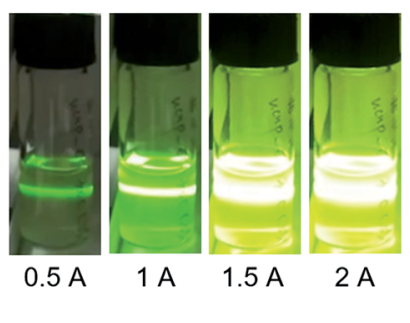

Fig. 2 The control of UCNP colour by changing the laser intensity. (a) Fluorescence peaks of UCNP excited with $980 \mathrm{~nm}$ laser. (b) The fluorescence colour change of UCNP from green to yellow according to the laser intensity from $0.5 \mathrm{~A}$ to $2 \mathrm{~A}$. 
adjusted by changing their compositions ${ }^{17-19}$ or temperature ${ }^{\mathbf{2 0 , 2 1}}$ in the synthetic steps. However, UCNP has the advantage to be colour-controlled by the excitation laser intensity due to several kinds of lanthanide ions which have multiple energy levels. This optical characteristics of UCNP is suitable to construct diverse colour fluorescence emitting on-off tattoo systems. After in vitro characterization, biocompatible HA-UCNP was assessed as an on-off tattoo system in 6 weeks old balb/c nude mice. HA-UCNP was topically administered onto the heart-shaped frame on the skin of nude mice for $40 \mathrm{~min}$. After complete washing, the fluorescence was visualized by $980 \mathrm{~nm}$ NIR laser irradiation which was collimated by the expanded lens (Fig. 3). Upon irradiating NIR light, the invisible heart pattern containing HAUCNP in the skin appeared as a new type of on-off tattoo

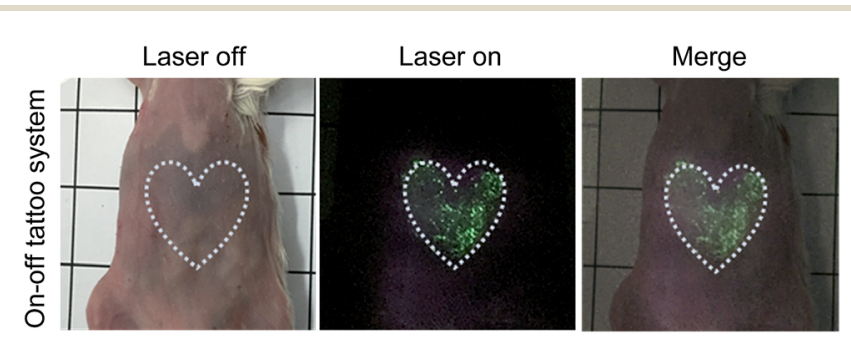

Fig. 3 In vivo on-off tattoo system which can be visualized in a controlled manner by irradiating $980 \mathrm{~nm}$ laser after transdermal delivery of HA-UCNP $(n=3)$.

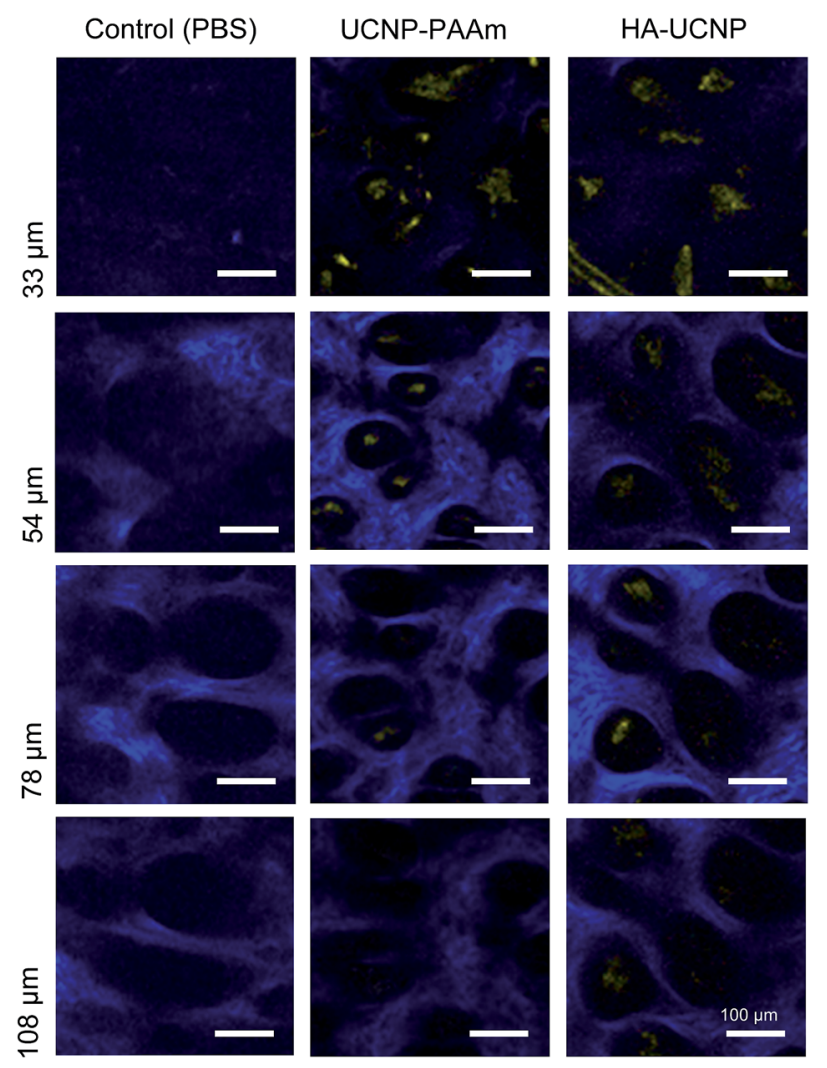

Fig. 4 Two-photon microscopic images of PBS, UCNP-PAAm and HA-UCNP transdermally delivered into the collagen layer of nude mice. system using non-invasive transdermal delivery and simple NIR light irradiation. The on-off tattoo system was also demonstrated in a triangle shape (Fig. S3, ESI $\dagger$ ). Animals were maintained in accordance with the guidelines of the Korea Food and Drug Administration, and all procedures with animals were approved by the Institutional Animal Care and Use Committee (IACUC) at the POSTECH Biotech Center.

Finally, two-photon microscopy was performed to assess the transdermal delivery of HA-UCNP. Fig. 4 shows two-photon microscopic images of PBS as a control, UCNP-PAAm and HA-UCNP topically delivered into the collagen layer of nude mice with $1050 \mathrm{~nm}$ laser excitation. The blue fluorescence represents collagen layers in the dermis by the second harmonic generation. ${ }^{9}$ Compared with UCNP-PAAm, a significant amount of HA-UCNPS were observed over the depth of $c a .100 \mu \mathrm{m}$ in the dorsal skin tissue. The transdermal delivery of UCNP-PAAm and HA-UCNP with increasing penetration depth was quantitatively compared by the two-photon microscopy (Fig. S4, ESI $\dagger$ ). The penetration spectra of HA-UCNP was shifted into the deeper depth than that of UCNP-PAAm. While positively charged UCNP-PAAm might be transdermally delivered by the nano size effect, HA-UCNP might be more effectively delivered than UCNP-PAAm through the skin by the facilitation of HA with a hydrophobic patch domain. In other words, HA can enhance the biocompatibility and the penetration of UCNP. Recently, UCNP has been widely investigated for multi-modal bioimaging ${ }^{22-24}$ and theranostic applications. ${ }^{25-27}$ Taken together, this platform technology might be successfully exploited for various biomedical applications.

\section{Conclusions}

HA-UCNP was successfully developed as a multi-colour on-off tattoo system. The conjugation of UCNP with HA was confirmed by DLS, FT-IR and HiLyte amine dye labelling. MTT assay revealed the improved biocompatibility of UCNP-PAAm after conjugation with HA. According to two-photon microscopy, HA-UCNP appeared to penetrate into the collagen layer of the dermis in nude mice. The fluorescence of invisible HA-UCNP patterned in a specific shape could be visualized by simple NIR laser irradiation. This new platform technology for on-off tattoo systems might be further applied to various photomedicines.

\section{Acknowledgements}

This research was supported by the Bio \& Medical Technology Development Program (No. 2012M3A9C6049791), Mid-career Researcher Program (No. 2015R1A2A1A15053779), and the Center for Advanced Soft-Electronics (Global Frontier Project, CASE-2015M3A6A5072945) of the National Research Foundation (NRF) funded by the Ministry of Science, ICT \& Future Planning, Korea.

\section{Notes and references}

1 J. V. Frangioni, Curr. Opin. Chem. Biol., 2003, 7, 626-634.

2 A. M. Smith, M. C. Mancini and S. Nie, Nat. Nanotechnol., 2009, 4, 710-711. 
3 S. Wang, L. Zhang, C. Dong, L. Su, H. Wang and J. Chang, Chem. Commun., 2015, 51, 406.

4 G. Chen, H. Qiu, P. N. Prasad and X. Chen, Chem. Rev., 2014, 114, 5161-5214.

5 H. S. Jung, K. S. Kim, S. H. Yun and S. K. Hahn, Nanomedicine, 2014, 9, 743-745.

6 I. C. M. Dea, R. Moorhouse, D. A. Rees, S. Arnott, J. M. Guss and E. A. Balazs, Science, 1973, 179, 560-562.

7 J. A. Yang, E. S. Kim, J. H. Kwon, H. Kim, J. H. Shin, S. H. Yun, et al., Biomaterials, 2012, 33, 5947-5954.

8 H. S. Jung, W. H. Kong, D. K. Sung, M. Y. Lee, S. E. Beack, D. H. Keum, K. S. Kim, S. H. Yun and S. K. Hahn, ACS Nano, 2014, 8, 260-268.

9 H. Lee, J. H. Lee, J. Kim, J. H. Mun, J. Chung, H. Koo, C. Kim, S. H. Yun and S. K Hahn, ACS Appl. Mater. Interfaces, 2016, 8, 32202-32210.

10 M. K. Gnanasammandhan, N. M. Idris, A. Kansal, K. Huang and Y. Zhang, Nat. Protoc., 2016, 11, 688-713.

11 L. Xia, X. Kong, X. Liu, L. Tu, Y. Zhang, Y. Chang, K. Liu, D. Shen, H. Zhao and H. Zhang, Biomaterials, 2014, 35, 4146-4156.

12 A. D. Ostrowski, E. M. Chan, D. J. Gargas, E. N. Katz, G. Han, P. J. Schuck, D. J. Milliron and B. E. Cohen, ACS Nano, 2012, 6, 2686-2692.

13 C. D. Laboda and C. L. Dwyer, Adv. Funct. Mater., 2016, 26, 2866-2874.

14 H. Lee, M. Y. Lee, S. H. Bhang, B. S. Kim, Y. S. Kim, J. H. Ju, K. S. Kim and S. K. Hahn, ACS Nano, 2014, 8, 4790-4798.
15 B. Zhou, B. Shi, D. Jin and X. Liu, Nat. Nanotechnol., 2015, 10, 924-936.

16 F. Wang, D. Banerjee, Y. Liu, X. Chen and X. Liu, Analyst, 2010, 135, 1797-2160.

17 Y. Lu, J. Zhao, R. Zhang, Y. Liu, D. Liu, E. M. Goldys, X. Yang, P. Xi, A. Sunna, J. Lu, Y. Shi, R. C. Leif, Y. Huo, J. Shen, J. A. Piper, J. P. Robinson and D. Jin, Nat. Photonics, 2014, 8, 32-36.

18 G. Tian, Z. Hu, L. Zhou, W. Yin, X. Liu, L. Yan, S. Jin, W. Ren, G. Xing, S. Li and Y. Zhao, Adv. Mater., 2012, 24, 1226-1231.

19 F. Wang and X. Liu, J. Am. Chem. Soc., 2008, 130, 5642-5643.

20 W. Niu, S. Wu, S. Zhang and L. Li, Chem. Commun., 2010, 46, 3908-3910.

21 S. Zheng, G. Ren, C. Xu and Q. Yang, CrystEngComm, 2011, 13, 1384-1390.

22 Z. Liu, F. Pu, S. Huang, Q. Yuan, J. Ren and X. Qu, Biomaterials, 2013, 34, 1712-1721.

23 Z. Liu, E. Ju, J. Liu, Y. Du, Z. Li, Q. Yuan, J. Ren and X. Qu, Biomaterials, 2013, 34, 7444-7452.

24 Z. Liu, K. Dong, J. Liu, X. Han, J. Ren and X. Qu, Small, 2014, 10, 2429-2438.

25 M. Sun, L. Xu, W. Ma, X. Wu, H. Kuang, L. Wang and C. Xu, Adv. Mater., 2015, 28, 898-904.

26 X. Wu, L. Xu, W. Ma, L. Liu, H. Kuang, N. A. Kotov and C. Xu, Adv. Mater., 2016, 28, 5907-5915.

27 S. Li, L. Xu, W. Ma, X. Wu, M. Sun, H. Kuang, L. Wang, L. A. Kotov and C. Xu, J. Am. Chem. Soc., 2016, 138, 306-312. 